\title{
Virtual meetings: a temporary choice or an effective opportunity for the future?
}

\author{
G. D'Anna ${ }^{1}$. F. D'Arco ${ }^{2}$ • J. Van Goethem ${ }^{3,4}$ \\ Published online: 22 May 2020 \\ (C) Springer-Verlag GmbH Germany, part of Springer Nature 2020
}

The ongoing SARS-CoV-2 pandemic that started in Wuhan, in the Chinese region of Hubei, lead to national lockdowns and closing of boundaries, affecting the academic and scientific world. Suddenly, the scientific community was forced to cancel all the planned international and national scientific meetings.

This is an unprecedented situation, especially for Europe, where the first outbreak occurred in Northern Italy, rapidly spreading to the rest of the continent and the whole world. But despite this, it is really interesting to note how quickly the scientific world reacted. With extensive use of the Internet, scientists united despite their confinement and restrictions imposed by new laws.

Instead of meeting at conferences in Europe and all around the globe there has been a switch to online webinars [1]. Once a niche market in scientific education and meetings, and usually for small groups only (such as research groups, journal clubs, etc.), these online virtual events suddenly expanded to large or very large audiences, immediately advancing webinars from a promising tool to a crucial scientific instrument of communication, primarily relying on what each person has in their pocket, a simple smartphone [2].

We are rediscovering how useful the front camera of our phones and laptops can be (beyond the selfies...), allowing us to meet, greet and see everyone despite being stuck at home. We can plan and initiate important events, even starting from one person's idea [3].

\section{G. D'Anna}

gennaro.danna@gmail.com

Radiology Unit, Humanitas Mater Domini, Castellanza, Varese, Italy

2 Radiology Department, Great Ormond Street Hospital, London, UK

3 Department of Biomedical Sciences, Antwerp University Hospital, Antwerp, Belgium

4 Department of Radiology, AZ Nikolaas, Sint-Niklaas, Belgium
A lecture series focused on pediatric neuroradiology, the "International Pediatric Neuroradiology Teaching Network" (https://aspnr.org/awards/international-pediatricneuroradiology-teaching-network/), conceptualized by Dr. Kshitij Mankad (London) and Dr. David Mirsky (Denver), sponsored by the American Society of Pediatric Neuroradiology (ASPNR), and endorsed by the European Society of Neuroradiology (ESNR), was so successful that some lectures were repeated up to three times in order to allow people from all timezones to attend.

Similarly, ESNR decided to organize a series of lectures as well, the ESNR WebLectures (https://www.esnr.org/en/esnrweb-lectures). This series consists of free weekly online lectures given by some of the top speakers in the field of Neuroradiology. This format is proving to be very successful as week after week the lecture attendance is at full capacity.

We are aware that there is a growing request for online educational resources and webinars offer many advantages as they conform to mandatory social distancing, make it easier for a speaker to reach a bigger audience, and allow users to receive information from the comfort and safety of his/her home. An extensive question and answer (Q\&A) session after a lecture is crucial to make the event more interactive and can be run smoothly by the event host (while Q\&As are generally limited to a few questions in live meetings), allowing clarification of some aspects not covered during the lecture or further elaboration on points of discussion.

On top of that, professional radiology study groups are flourishing on messaging apps (such as Telegram or the UK app Pando, specifically designed for secure data sharing among physicians) and allow doctors from all over the world to share difficult cases and interesting scientific papers.

On the other hand, the lack of human contact should not be underestimated, especially the value of professional networking and the importance of personal interaction. Moreover, webinars are also exposed to all known online safety issues, such as network or hardware failures, hackers' attacks now known as "zoombombing", and other IT safety concerns. 
Even sharing anonymized images on open messaging groups has, depending on country, important privacy issues.

Saying that, the real value of this "online education revolution" is that rare diseases, unusual radiological patterns, and complex cases that before were only seen by a neuroradiologist once in a lifetime, now can benefit of the experience of, literally, the whole world, making patient care more efficient and knowledge readily available to individuals that can't easily travel to attend in-person meetings.

In conclusion, even a serious global emergency like the COVID19 pandemic can help us explore new ways of interaction and communication when we are forced to stay at home. We will see if this medium will be a temporary choice or will develop into an organized tool for teaching and patient care. We think it is an opportunity not to be missed. Its future use could be many, alongside "in person" meetings, that will probably still remain indispensable for sharing culture and knowledge.

\section{References}

1. Chou CL, Promes SB, Souza KH, Topp KS, O'Sullivan PS (2012) Twelve tips for facilitating successful teleconferences. Med Teach 34(6):445-449. https://doi.org/10.3109/0142159X.2012.668241

2. Pinzon-Perez H, Zelinski C (2016) The role of teleconferences in global public health education. Glob Health Promot 23(2):38-44. https://oi.org/10.1177/1757975914567180

3. Knipfer C, Wagner F, Knipfer K et al (2019) Learners' acceptance of a webinar for continuing medical education. Int J Oral Maxillofac Surg 48(6):841-846. https://doi.org/10.1016/j.ijom.2018.11.010

Publisher's note Springer Nature remains neutral with regard to jurisdictional claims in published maps and institutional affiliations. 